\title{
Von umstrittenen Pionierprojekten zum Erfolgsmodell
}

Arbeitsfeld Niederschwellige Arbeit

Franziska Schicker ${ }^{1}$, Natascha Inauen ${ }^{2}$

\section{Das Arbeitsfeld der niederschwelligen Sozialarbeit mit substanz- abhängigen Personen}

Nach Jahren erfolgloser, repressiver Drogenpolitik und den Erfahrungen mit den offenen Drogenszenen in vielen Städten der (Deutsch-) Schweiz als bekanntestes Beispiel sind der Platzspitz und der Letten in Zürich zu erwähnen - wurde in den 1990er-Jahren die Vier-Säulen-Drogenpolitik schweizweit eingeführt. In enger Zusammenarbeit entwickelten Fachleute und Politiker das Modell der vier Säulen. Das bisherige Drei-Säulen-Modell, bestehend aus Prävention, Therapie und Repression, wurde mit der vierten Säule Überlebenshilfe (später Schadensminderung) ergänzt.

Im Zuge dieser Entwicklung wurden die ersten Drogeninjektionsräume und Spritzentauschprojekte, aus denen später die heutigen Kontakt- und Anlaufstellen (K\&A) entstanden, eröffnet sowie weitere niederschwellige Projekte im Bereich Gesundheit, Wohnen, Arbeit und Betreuung umgesetzt. Diese Säule umfasst alle Strategien und Massnahmen zur Minderung der negativen Folgen des Drogenkonsums für die Konsumierenden sowie die Gesellschaft.

Die K\&A bieten substanzkonsumierenden Erwachsenen niederschwelligen Zugang zu schadensmindernden Angeboten und Beratung. Interdisziplinäre Teams, bestehend aus Sozialarbeitenden und medizinischem Fachpersonal (Psychiatrie und Somatik) unterstützen die Klientel in ihrem, von der Substanzabhängigkeit bestimmten, Alltag. Die Klientel kann in beaufsichtigten Injektions- und Inhalationsräumen selber mitge-

1Sozialarbeiterin FH B.Sc, Kontakt- und Anlaufstellen Stadt Zürich.

2 Sozialarbeiterin FH M.Sc, Case Managerin, Leiterin Beratung chronisch Substanzabhängiger im niederschwelligen Arbeitsfeld, Zürich (bis Okt. 2019). 
brachte (illegale und/oder verschreibungspflichtige legale) Substanzen unter hygienischen Bedingungen konsumieren. Ziel und Auftrag des Angebotes ist es, die Übertragung von Infektionskrankheiten wie HIV und Hepatitis zu verringern, die Zahl von Todesfällen durch Überdosierung zu reduzieren, die soziale Integration und Lebensqualität zu verbessern sowie den öffentlichen Raum zu entlasten.

\subsection{Rechtliche Verankerung}

Die gesetzliche Legitimation der K\&A basiert auf den vier national verankerten Säulen der schweizerischen Drogenpolitik (Prävention, Therapie, Schadensminderung und Repression). Die Revision des Bundesgesetzes über die Betäubungsmittel und psychotropen Stoffe (BetmG) ${ }^{3}$ wurde durch das Stimmvolk im Jahr 2008 angenommen und verpflichtet seither die Kantone explizit, Angebote der Schadensminderung zu schaffen (Art. $3 g$, BetmG).

Angebote der Schadensminderung werden durch öffentliche sowie privaten Institutionen zur Verfügung gestellt, wobei Organisationsform, Grösse, Standorte sowie Anzahl Mitarbeitende variieren können.

\subsection{Finanzierung und Ressourcenallokation}

Die Leistungen, welche die Klientel in den niederschwelligen Institutionen bezieht, werden durch die öffentliche Hand und/oder private Trägerschaften finanziert. Die Finanzierung durch die öffentliche Hand unterliegt wiederkehrenden Leistungsüberprüfungen und möglichen Sparmassnahmen. So wurden im Jahr 2016 die kantonalen Beiträge an die Stadt Zürich für die dezentrale Drogenhilfe gestrichen (vgl. Medienmitteilung Finanzdepartement Stadt Zürich 2016). Seither werden die Kosten für die drei Kontakt- und Anlaufstellen der Stadt Zürich vollumfänglich von der Gemeinde getragen.

\footnotetext{
${ }^{3}$ BetmG: www.tinyurl.com/jmy8yaq, Zugriff 28.12.2019.
} 


\subsection{Politische Positionierung}

Die Notwendigkeit von Kontakt- und Anlaufstellen mit Konsumraum galt von der Gesellschaft und den politischen VertreterInnen aufgrund des «Platzspitztraumas» lange als unbestritten. Die katastrophalen Zustände der suchtmittelabhängigen Menschen war in den 1990er-Jahren für die breite Bevölkerung sichtbar und hat die Politik dazu veranlasst, eine nachhaltige Lösung zu finden.

Erst erfolgte 1992 die repressive Räumung des Platzspitzareals, welche jedoch ohne Begleitmassnahme der Schadensminderung die nächste offene Drogenszene am Bahnhof Letten in Zürich zur Folge hatte. Man realisierte, dass eine Schliessung der offenen Drogenszene ohne niederschwellige Einrichtungen wie Kontakt- und Anlaufstellen, wohl wieder zu einer Verschiebung des Elends führen würde. Heute ist der Nutzen der K\&A für Aussenstehende nicht mehr so deutlich ersichtlich wie damals. Die Situation der Klientel hat sich durch die Vielfalt an suchtspezifischen Angeboten stark verbessert. Doch wenn das Problembewusstsein in der Bevölkerung und somit der Politik schwindet, besteht die Gefahr, dass bestehende Angebote in Frage gestellt und deren Finanzierung ${ }^{4}$ gestrichen werden. Somit können schadensmindernde Angebote ohne ausreichende Öffentlichkeitsarbeit Opfer ihres eigenen Erfolges werden.

\subsection{Ausbildung und Zugang zum Arbeitsfeld}

Innerhalb des Arbeitsfeldes verfügen die Mitarbeitenden über vielfältige Bildungshintergründe und nebst ihrer Grundausbildung über zusätzliche Aus- und Weiterbildungen. Die Organisation beteiligt sich zudem finanziell an Aus- und Weiterbildungen, welche für das Arbeitsfeld von Nutzen sind. Für alle Mitarbeitenden ist nur der rein medizinische Basic Life Support für Erste Hilfe vor Ort Pflicht.

\footnotetext{
${ }^{4}$ Medienmitteilung Finanzdepartement Stadt Zürich vom 31.08.2016: www.tinyurl.com/smbe9us, Zugriff 28.12.2019.
} 


\subsection{Menschenbild im Arbeitsfeld}

Das Menschenbild ist geprägt von einer humanistischen Grundhaltung. In dieser wird anerkannt, dass der Mensch danach strebt, sein Leben sinnhaft und selbstbestimmt zu führen, und dass alle Menschen gleichwertig und gleichberechtigt in ihrer Würde sind. Für die Arbeit mit Menschen heisst dies, dem Gegenüber mit Respekt und auf Augenhöhe zu begegnen und im Sinne einer akzeptierenden Haltung keine Wertung der Lebensführung vorzunehmen. Konsum und Genuss von psychoaktiven Substanzen werden als Teil der menschlichen Lebensweise betrachtet.

\section{Soziale Arbeit im niederschwelligen Setting mit Substanzabhän- gigen}

\section{$2.1 \quad$ Funktion der Sozialen Arbeit}

Die Soziale Arbeit ist in den K\&A hauptsächlich für die individuelle, weiterführende KlientInnenarbeit (sozialarbeiterische Begleitung und Unterstützung über das Angebot der K\&A hinaus), für die Konzeptarbeit sowie für die Netzwerkarbeit verantwortlich. Die Tätigkeiten entsprechen somit eher der klassischen Sozialen Arbeit und nicht dem Profil der SozialpädagogInnen oder GemeinwesenarbeiterInnen. Die Soziale Arbeit ist in diesem Arbeitsfeld zudem die Schnittstelle zu anderen Fachdisziplinen wie beispielsweise Medizin, Psychologie, Recht und Soziologie. Sie arbeitet mit unterschiedlichen Modellen und Methoden dieser Fachdisziplinen und adaptiert diese gezielt und praxisorientiert für ihr Arbeitsfeld.

\subsection{Angebote der Sozialen Arbeit}

Wie bereits erwähnt, können in Kontakt- und Anlaufstellen mitgebrachte, Substanzen im geschützten Rahmen konsumiert werden, wobei jedoch nicht alle Kontakt- und Anlaufstellen der Schweiz über Konsumräume verfügen. In Zürich gibt es drei Standorte mit je einem Raum für das Inhalieren und Injizieren von psychoaktiven Substanzen. Die Klientel hat die Möglichkeit, zu duschen, sich neu einzukleiden und eine Mahlzeit zu sich 
zu nehmen. Wasser und Tee werden gratis abgegeben, alle anderen Getränke und Mahlzeiten werden zum Selbstkostenpreis verkauft. Die Kontakt- und Anlaufstellen dienen auch als Aufenthaltsort und es besteht die Möglichkeit, soziale Kontakte zu knüpfen. Die Konsumräume werden vom Personal überwacht, um bei medizinischen oder sozialen Notfällen sofort intervenieren zu können. Die Standards der Kontakt- und Anlaufstellen sind gesamtschweizerisch festgelegt und online zugänglich (vgl. Infodrog/Fachverband Sucht 2011).

Für Fragestellungen der Mitarbeitenden aus dem Arbeitsalltag stehen Methoden wie extern begleitete Supervisionen, interne Kollegiale Beratungen (z. B. Tietze 2010) oder interne Intervisionen (z. B. Lippmann 2013) zur Verfügung. Um die Qualität der Arbeit zu fördern, werden Arbeits- und Fachgruppen zu unterschiedlichen Themen gebildet, welche interne Standards erarbeiten und einführen. Diese Themen sind jedoch nicht spezifisch der Sozialen Arbeit zuzuordnen.

\subsection{Interventionsmodelle}

Für die Gestaltung des alltäglichen Zusammenseins in den Konsum- und Aufenthaltsräumen gelten wenige, dafür klare Hausregeln (bspw. Gewaltfreiheit), welche mit Sanktionen in Form von möglichst kurzen Hausverboten durchgesetzt werden. Im Akutfall werden Sanität, Notfallpsychiatrie und/oder Polizei beigezogen.

Es bestehen national unterschiedliche Interventionsmodelle. In den Kontakt- und Anlaufstellen Zürich zum Beispiel ist die weiterführende sozialarbeiterische Begleitung, für die Klientel freiwillig, ausser bei betreuungspflichtigen Eltern oder jungen Erwachsenen unter 25 Jahren. Hierfür besteht ein Bezugspersonensystem, für das sich alle Mitarbeitenden zur Verfügung stellen. Für zeitintensivere oder komplexere Anliegen besteht die Möglichkeit, sich an ein intern über alle Angebote im Arbeitsfeld vernetztes Team zu wenden, welches niederschwellige, subsidiär ausgerichtete Sozialberatung anbietet. Dieses Team besteht ausschliesslich aus SozialarbeiterInnen FH oder aus Psychiatriepflegefachpersonen FH mit sozialarbeiterischer Zusatzausbildung.

Die niederschwellige Sozialberatung wird direkt vor Ort angeboten wodurch die Mitarbeitenden der Klientel bekannt und somit vertraut sind. 
Die Beratenden sind unter Berücksichtigung des Datenschutzes mit anderen Angeboten via Fallarbeit gut vernetzt und begleiten bei Bedarf die Klientel auch an Termine. In der niederschwelligen Sozialberatung ist von klassischer Sozialarbeit die Rede, welche administrativ und subsidiär ausgerichtet ist. Entscheidend für die Arbeitsweise sind individuelle Spezialisierungen bspw. auf Sozialversicherungsrecht oder motivierende Gesprächsführung sowie die persönliche Arbeitserfahrung der Mitarbeitenden.

Im Arbeitsfeld gibt es verschiedene Herangehensweisen in der Sozialen Arbeit. Als mögliche Methodik ist das Case Management (CM) nach Wendt (2001) zu erwähnen, welches je nach Struktur der Institution auf unterschiedlichen Ebenen umgesetzt werden kann. Grundsätzlich systematisiert das CM die Fallführung anhand eines Regelkreises (Intake, Assessment, Zielesetzung und Handlungsplanung, Monitoring, Evaluation, Reassessment/Abschluss) und führt eine Aktenführung nach sozialarbeiterischen Standards. Im Gegensatz zur heutigen Aktenführung wurden früher im Arbeitsfeld, begründet mit der Niederschwelligkeit und der Anonymität der Klientel, weniger Daten erfasst. Das CM arbeitet koordiniert und unter Einhaltung des Datenschutzes mit dem gesamten Helfernetz der Klientel zusammen und kann so einerseits kostenintensive Doppelspurigkeiten und Leerläufe vermeiden und andererseits die teils für die Klientel unübersichtlichen Zuständigkeiten auf eine Ansprechperson reduzieren. Das CM ist über das eigene Arbeitsfeld hinaus vernetzt und steht so im stetigen Austausch zu anderen AkteurInnen aus dem Sozialbereich. Neu generiertes Fachwissen fliesst so über die Vernetzung oder die KlientInnenarbeit in Form von Standards, Konzepten und Weiterbildungen wieder zurück in die Organisation und sichert so eine Arbeitsweise nach aktuellen Standards.

Ergänzend zu Schadensminderungsangeboten und Beratung wird in den K\&A zunehmend mit Konsumreduktionsprogrammen gearbeitet. Diese zieloffene Soziale Arbeit ist nicht abstinenzorientiert und bietet Menschen mit Abhängigkeitserkrankungen die Möglichkeit, an der Veränderung ihres Substanzkonsums zu arbeiten, und zwar auf das Ziel hin, welches sie sich selbst setzen. 
Die Klientel kann an einem KISS-Programm ${ }^{5}$ teilnehmen, welches von Mitarbeitenden mit entsprechender Trainerausbildung angeboten wird. KISS steht für «Kompetenz im selbstbestimmten Substanzkonsum» und ist ein verhaltenstherapeutisches Selbstmanagementprogramm, welches aus zwölf strukturierten Sitzungen besteht. Teil dieser Sitzungen sind u. a. das Führen eines Konsumtagebuches, das Festlegen von wöchentlichen Konsumzielen, das Erkennen und Bewältigen von Risikosituationen.

Bei den Sozialarbeitenden muss die Bereitschaft vorhanden sein, offensiv und sanktionsfrei mit der Klientel über verschiedene Konsumziele ins Gespräch zu kommen. Basis dazu bildet die Motivierende Gesprächsführung (Motivational Interviewing MI). Motivierende Gesprächsführung bezeichnet einen «kooperativen Gesprächsstil, mit dem wir einen Menschen in seiner eigenen Motivation zur und seinem eigenen Engagement für Veränderung stärken können» (Miller/Rollnick 2015: 27).

\subsection{Beziehungsarbeit}

Die kontinuierliche Beziehungspflege und die daraus resultierende Interventionsberechtigung gelten als Grundlage aller gelingenden Massnahmen. Dies ermöglicht den Mitarbeitenden, in prekären Alltagssituationen deeskalierend einzugreifen. Zudem wird über die regelmässige Beziehungspflege eine Vertrauensbasis geschaffen, welche es der Klientel erleichtert, innerhalb einer Beratung belastende und schambeladene Themen anzusprechen und sich auf eine freiwillige Unterstützung einzulassen.

Dennoch ist die Beziehung zwischen der Klientel und den Sozialarbeitenden im Sinne von Kaspar Geiser (2004: 235) vertikal, also in einem Machtgefälle strukturiert. Die Mitarbeitenden nehmen auch die Rolle der Kontroll- und Entscheidungsinstanz ein (vgl. ebd.) und sorgen dafür, dass die K\&A für alle NutzerInnen gleichermassen als Schutz- und Rückzugsraum dient. Dies bedeutet im konkreten Fall, die Umsetzung und Durchsetzung der Hausordnung, der medizinischen Anordnungen sowie von Auflagen und Sanktionen.

\footnotetext{
${ }^{5}$ Vgl. www.kiss-heidelberg.de, Zugriff 28.12.2019.
} 
Um den Beziehungsaufbau zur Klientel im Arbeitsalltag zu fördern, wird eine akzeptierende und wertefreie Haltung gegenüber der Lebensführung der Klientel vertreten, in der nicht die Abstinenz, sondern die individuelle Lebensqualität im Vordergrund steht. Im Arbeitsfeld wird das Du zur Klientel gepflegt und das Zeitnehmen für alltägliche Gespräche ohne sozialarbeiterischen Inhalt gehört zum aktiven Beziehungsaufbau. Nicht zur anerkannten und professionellen Beziehungspflege gehören private Kontakte ausserhalb der Arbeitszeit, Angaben von privaten Adressen der Mitarbeitenden oder der Austausch von privaten Gütern (Geschenke, Geldgaben etc.).

\subsection{Stellenwert der Sozialen Arbeit}

Die Soziale Arbeit wird in der niederschwelligen Arbeit als eigene Profession mit ihren eigenen Aufgaben, Arbeitsweisen und Interessen anerkannt. Dies jedoch als Einheit ohne Unterteilung in Sozialarbeitende, SozialpädagogInnen und GemeinwesenarbeiterInnen. Die Soziale Arbeit fokussiert sich auf die Arbeit mit und für die Klientel und nimmt innerhalb der interdisziplinären Fallarbeit eine wichtige Rolle bei der Koordination aller Beteiligten und von deren Dienstleistungen ein. Zunehmend rückt der Fokus der Sozialen Arbeit auf die Legitimierung des Arbeitsfeldes, indem sie die Wirkung und den Nutzen der Dienstleistungen der K\&A auch auf politischer Ebene und gegenüber den Geldgebenden darlegt und transparent macht.

Innerhalb der Institution ist der Autonomiegrad der Sozialen Arbeit eher hoch einzustufen, sie ist jedoch stets an den Auftrag der eigenen Institution gebunden. Der Auftrag der Sozialen Arbeit ist abhängig von der aktuellen politischen Situation, von gesellschaftlichen Trends und der Kostendeckung der finanzierenden Stellen wie Krankenkassen, wirtschaftliche Sozialhilfe oder den Ergänzungsleistungen zur IV/AHV.

Konzeptionell verankert ist die Soziale Arbeit in den K\&A nur in Bezug auf die weiterführende KlientInnenarbeit. Das heisst, es werden in der Beratung der Klientel Methoden und Standards der Sozialen Arbeit angewendet. Dies betrifft die Situationserfassung, Gesprächs- und Aktenführung. Es ist institutionsabhängig, ob konzeptuell verankert ist, dass bei der 
Teamzusammensetzung auf ein ausgewogenes Verhältnis zwischen sozialarbeiterisch, somatisch und psychiatrisch ausgebildetem Personal geachtet wird oder nicht.

\subsection{Entwicklung der Professionalität}

Soziale Arbeit ist historisch betrachtet aus der Freiwilligenarbeit entstanden. Im Laufe der Jahre haben sich professionelle Arbeitsstandards etabliert, die jedoch immer wieder von unterschiedlichen Akteuren aus Politik und Gesellschaft in Frage gestellt werden. Im Zuge von Sparmassnahmen wird erneut der Einsatz von Freiwilligenarbeit in Betracht gezogen. Diese hat selbstverständlich ihre Berechtigung, muss jedoch gegenüber der Sozialen Arbeit gerade im niederschwelligen Bereich kritisch betrachtet werden. Die Soziale Arbeit in den K\&A wurde einerseits geprägt von charismatischen Führungsfiguren, andererseits von Freiwilligen, die ohne professionelle Standards der Gefahr des Rollenkonfliktes ausgesetzt sind (z. B. beim Durchsetzen von Regeln). Die Vergangenheit hat gezeigt, dass in einem Klima von struktureller Hierarchie und emotionaler Nähe immer wieder Übergriffe aller Art auf die Schutzbedürftigen stattgefunden haben.

Professionelle Standards sind vor allem aus zwei Gründen äusserst wichtig. Zum einen legen sie fest, in welchem Umfang die Arbeit mit der Klientel zu erfolgen hat. Zum anderen fördern die zu solchen Standards gehörenden Supervisionen und kollegiale Beratungen die Selbstreflexion in Bezug auf die eigene Arbeit, und stellen so einen wichtigen Teil professionellen Arbeitens dar.

\subsection{Angrenzende Arbeit}

In den Anfängen der Schadensminderung war das Arbeitsfeld sehr stark medizinisch ausgerichtet, da der Gesundheitszustand der Klientel oft prekär war und die Betroffenen keine adäquate medizinische Versorgung erhielten. Der Zugang zur medizinischen Versorgung für die Klientel hat sich mit der Einführung der obligatorischen Grundversicherung 1996 in der Schweiz stark verbessert. Somit ging die Entwicklung im Arbeitsfeld 
weg von der reinen Erbringung medizinischer Leistungen hin zur Koordination verschiedener Leistungen. Heute fokussieren sich medizinisch ausgebildete Mitarbeitende vermehrt auf die Erbringung von Leistungen wie basismedizinische Versorgung sowie auf das Einhalten von medizinischen Standards, während sich Mitarbeitende mit sozialarbeiterischem Hintergrund vermehrt auf Bereiche wie weiterführende KlientInnenarbeit, Konzeptarbeit, Vernetzung und Legitimierung fokussieren.

\section{Interdisziplinäre Zusammenarbeit}

\subsection{Beteiligte Professionen}

Heute wird von einer klar multiperspektivischen Herangehensweise gesprochen. Jede Profession leistet ihren Beitrag zum Hilfesystem, womit ein Netz um die Klientel entsteht. Die Zusammenstellung der Beteiligten in einem Helfernetz hängt von der Problemstellung des Individuums ab. Die Soziale Arbeit nimmt darin eine formell verankerte, koordinierende und planende Rolle innerhalb des Helfernetzes ein, ohne die eine Zusammenarbeit über alle beteiligten Akteure hinweg nicht möglich wäre.

\subsection{Strukturierung der interdisziplinären Zusammenarbeit}

Die Zusammenarbeit zwischen den Professionen wie bspw. den medizinischen und sozialarbeiterischen Fachkräften innerhalb der Institution funktioniert aufgrund ihrer gemeinsamen Geschichte im Arbeitsfeld und aufgrund des gemeinsamen Auftrages sehr gut.

Auf transparente Zusammenarbeit und Kommunikation unter den unterschiedlichen AkteurInnen sowie mit der Klientel wird grosser Wert gelegt. VertreterInnen aller vier Säulen der Schweizer Drogenpolitik werden innerhalb von Fallkonferenzen mit in die Planung einbezogen. Auf die gute Zusammenarbeit mit der Polizei wird viel Gewicht gelegt, da das Verhältnis aus historischen und gesetzlichen Gründen in der niederschwelligen Arbeit mit Substanzabhängigen nicht immer einfach war. Professio- 
nelle Sozialarbeitende betreiben keine einseitige Parteinahme für die Klientel, sondern fördern aktiv die stetige Verbesserung interdisziplinärer Zusammenarbeit.

Interdisziplinäre Zusammenarbeit findet auch in Fachgruppen und Fachverbänden auf regionaler und nationaler Ebene statt. Hier zu erwähnen ist allen voran der Fachverband Sucht, in welchem sich verschiedene Fachgruppen vernetzen (Kontakt-und Anlaufstellen, Prävention, Substitution etc.). Der Fachverband Sucht setzt sich für eine menschenwürdige und fachlich fundierte Drogenpolitik ein und vertritt die Interessen der Mitglieder gegenüber Gesellschaft und Politik.

\section{Stand der Professionalisierung der Sozialen Arbeit im nieder- schwelligen Suchtbereich}

\subsection{Expertise und Profession der Sozialen Arbeit}

Die Soziale Arbeit beansprucht im niederschwelligen Setting keine zusätzliche spezifische eigene Expertise, benötigt wird jedoch suchtmedizinisches Wissen sowie Kenntnisse über die Lebenswelt der Betroffenen. Dies liegt in der Entstehung des Arbeitsfeldes begründet. Die Pionierphase nach Glasl/Kalcher/Piber (2014) in den 1980er- und 1990er-Jahren war geprägt durch unterschiedlichste meist persönlich motivierte AkteurInnen, welche die prekäre gesundheitliche und soziale Situation der Abhängigen, sowie die Wirkungslosigkeit der damals rein repressiven, staatlichen Interventionen scharf kritisierten und öffentlich thematisierten. Mit zunehmender Erfahrung und genauerer Kenntnis der abhängigen Menschen und ihrer Lebenswelt professionalisierte sich das Arbeitsfeld schrittweise und die Soziale Arbeit gewann an Bedeutung. Die Erkenntnis, dass substanzabhängige Personen oft auch im zwischenmenschlichen Bereich Abhängigkeitsmuster ausleben und dies auch zwischen Mitarbeitenden und NutzerInnen der K\&A zu Abgrenzungsproblemen führt, hatte beispielsweise zur Folge, die Nähe-Distanz-Thematik so stark zu gewichten, wie wir es heute tun. Insbesondere diese Thematik ist mit ein Grund, warum das Arbeitsfeld nach Mitarbeitenden mit geeignetem beruflichen Hintergrund wie Sozialarbeit, Psychiatrie und Somatik verlangt. 


\subsection{Positionierung und Identität der Sozialen Arbeit}

Die weiterführende KlientInnenarbeit gehört zu den Kernaufgaben der Sozialen Arbeit in diesem Arbeitsfeld, das heisst: Wird innerhalb eines Falls mit anderen Institutionen zusammengearbeitet, findet ein Informationsaustausch meist zwischen den Sozialarbeitenden statt, welche ihre Institution vertreten oder für die betroffene Klientel zuständig sind. Somit wird die Soziale Arbeit auch zur Schnittstellenkoordinatorin.

\section{Bestandsaufnahme und Ausblick}

\subsection{Herausforderungen für das Arbeitsfeld}

Grundsätzlich ist es für die Soziale Arbeit schwierig, ihre Wirkung anhand von Zahlen zu belegen. Hinzu kommt, dass mit zunehmendem Erfolg die Berechtigung für die eigene Existenz schwindet. Somit muss belegt werden, dass sich die Situation bei einer Aufhebung der Angebote wieder verschlechtern würde, was jedoch erst eine Hypothese ist. Ausserdem ist der Grundgedanke der Schadensminderung der bestmögliche Umgang mit schon entstandenem Schaden. Mit der Entwicklung im Bereich der Psychiatrie, wo Behandlungen vermehrt vom stationären auf den ambulanten Sektor verlagert werden (vgl. Schweizerisches Gesundheitsobservatorium 2010: 1), könnte sich auch die Klientel der K\&A ändern. Gehörten Personen mit komorbiden Störungen schon immer mit zur Klientel der K\&A, könnten sie in Zukunft eine der Hauptgruppen ausmachen. Somit fände eine Verlagerung des medizinischen verlangten Fachwissens von der Somatik (Wundpflege etc.) hin zur Psychiatrie (Wissen über weitere psychiatrische Krankheitsbilder neben der Abhängigkeit) statt. Für die Soziale Arbeit bedeutet dies, dass sie ihre Kooperationen mit dem Arbeitsfeld der Psychiatrie ausbaut, um die fachlichen Kompetenzen der Mitarbeitenden entsprechend zu erweitern.

Eine Herausforderung in der niederschwelligen Arbeit mit substanzabhängigen Personen wird sicherlich die theoretisch-konzeptuelle Einbettung sein. Ein grosser Teil der täglichen Arbeit wurde über die Jahre im Sinne einer «Best Practice» entwickelt und hat sich bewährt. Indessen fehlt 
teilweise der theoretische Bezugsrahmen, was die professionelle Sozialarbeit in diesem Arbeitsfeld angreifbar macht.

Eine weitere Herausforderung besteht darin, die professionelle Arbeit der interdisziplinären Berufsgruppen der K\&A zu fördern und sich auf eine gemeinsame Haltung zu einigen sowie das eigene Handeln zu reflektieren und kritisch zu hinterfragen.

Aufgrund des allgemeinen Kostendrucks in der Sozialen Arbeit gibt es zunehmend Tendenzen, Angebote wie Supervisionen für die Teams und die einzelnen MitarbeiterInnen zu streichen. Supervisionen, Coachings für Sozialarbeitende und Führungskräfte leisten jedoch einen wichtigen präventiven Beitrag um vermehrte, teilweise lange Krankheitsausfälle zu reduzieren.

Sozialarbeitende verfügen meist über eine hohe intrinsische Motivation und sehen einen grossen Sinn in der Arbeit mit der Klientel. Mit zunehmendem finanzpolitischem Legitimationsdruck steigen die Anforderungen, die Wirksamkeit der Arbeit mit der Klientel mittels Kennzahlen zu belegen.

Wie vorgängig erwähnt, verändert sich die Klientel in der niederschwelligen Sozialen Arbeit mit Abhängigen. Die grösste Gruppe der KlientInnen besteht zunehmend aus Personen mit einer starken psychischen Beeinträchtigung. Dieser Umstand erfordert von den Sozialarbeitenden ein hohes Mass an Empathie und Akzeptanz für das Gegenüber, um adäquat auf dessen Bedürfnisse eingehen zu können. Diese Haltung und Kompetenz lässt sich schwer bis kaum erfassen und abbilden, und doch ist sie zentral für eine gelingende Zusammenarbeit mit der Klientel.

\subsection{Bildungsarbeit für die Zukunft}

Angebote der Säule Schadensminderung haben ihren Ursprung in den offenen Drogenszenen der 1980er- und 1990er-Jahre. Erst mit veränderten Gesetzen (die Abgabe von sterilem Injektionsmaterial wurde 1985 durch den Zürcher Kantonsarzt verboten, das Verbot kurz darauf wieder aufgehoben) und dem Willen der unterschiedlichsten Akteure zur Zusammenarbeit konnten Angebote geschaffen werden, wie wir sie heute kennen. K\&A sind das direkte Resultat der damaligen enorm innovativen und pragmatischen Zusammenarbeit auf verschiedenen Ebenen. 
Mit schadensmindernden Angeboten konnte die Ausbreitung von HIV/Aids und anderen übertragbaren Krankheiten wie Hepatitis $C$ bei substanzkonsumierenden Personen drastisch reduziert werden. Diese Tatsache ist von grosser Bedeutung für die Gesamtbevölkerung. Erinnern wir uns nur an den, damals epidemischen, Anstieg von HIV-Ansteckungen und die grosse Angst der QuartierbewohnerInnen vor herumliegenden Spritzen in Innenhöfen, auf Toiletten und Spielplätzen.

Damit dieser historische Kontext nicht in Vergessenheit gerät, ist es notwendig, dass Fachleute mit ihrem spezifischen Wissen gesellschaftlich und politisch aktiv bleiben oder werden. Fachleute aus der Sozialen Arbeit müssen sich in Debatten einmischen und dürfen die Definitionshoheit für ihr Arbeitsfeld nicht aus den Händen geben.

\subsection{Internationale Zusammenarbeit}

Das Schweizer Vier-Säulen-Modell hat sich enorm bewährt und steht für einen grossen Erfolg im Umgang mit Menschen mit einer Substanzabhängigkeit. Zunehmend interessieren sich daher Staaten auf der ganzen Welt für das erfolgreiche Schweizer Modell, was sich darin widerspiegelt, dass immer wieder Delegationen der unterschiedlichsten Länder Institutionen der Säule Schadensminderung besichtigen, allen voran die K\&A.

In der Schweizer Bevölkerung und Politik ist indessen das Bewusstsein für dieses erfolgreiche Modell am Schwinden. Daher ist es eine grosse Chance und Anerkennung, anderen Institutionen und Staaten mit ExpertInnenwissen zur Seite zu stehen. Die internationale Vernetzung der unterschiedlichsten Akteure der Schadensminderung schreitet zwar stetig voran (v. a. via Social Media), könnte jedoch noch aktiver von Führungskräften und politischen AmtsträgerInnen gefördert werden.

\subsection{Plädoyer für die Schadensminderung}

Schadensminderung ist das letzte Auffangbecken, wenn die Prävention nichts abwenden, die Repression nicht abschrecken und die Therapie keinen nachhaltigen Weg aufzeigen konnte.

Die Existenz der Säule Schadensminderung ist das Ergebnis einer pragmatischen und fortschrittlichen Suchtpolitik. Sie widerspiegelt die 
Akzeptanz innerhalb der Gesellschaft gegenüber Menschen, welche trotz vielfältiger Hilfsangebote nicht fähig oder willens sind, aus der Abhängigkeit auszusteigen. Dennoch steht diese Säule wiederholt bei unterschiedlichen politischen Parteien in der Kritik. Je mehr die Erinnerung an die offenen Drogenszenen verblasst, desto mehr finden wieder Stimmen Gehör, welche die gemachten Erfahrungen verkennen und die immense positive Wirkung schadensmindernder Angebote nicht als solche wahrnehmen. Somit hat die Soziale Arbeit in diesem Arbeitsfeld mehrere wichtige Aufgaben zugeteilt bekommen. Sie kümmert sich nicht nur um die KlientInnen- und Konzeptarbeit, sondern sie vertritt die Bedürfnisse der Klientel auf politischer und ethischer Ebene und setzt sich somit für eine Drogenpolitik im Dienste der gesamten Gesellschaft ein.

Angebote der Schadensminderung sind aufgrund ihrer Thematik auf Imageebene eher schwierig positiv zu besetzen. Das muss nicht für immer so sein, gerade wenn man bedenkt, wie - wie oben erwähnt - die Angebote erheblich dazu beigetragen haben, die Lebensqualität der unterschiedlichsten Menschen zu steigern. Einerseits profitiert natürlich die Klientel von den Angeboten, andererseits erfahren auch die QuartierbewohnerInnen und schlussendlich die gesamte Bevölkerung den Nutzen und die Wirkung der Institutionen im niederschwelligen Bereich.

\section{Literatur}

Geiser, K. (2004): Problem- und Ressourcenanalyse in der Sozialen Arbeit. Eine Einführung in die Systemische Denkfigur und ihre Anwendung. Luzern.

Glasl, F./Kalcher, T./Piber, H. (2014): Professionelle Prozessberatung Das Trigon-Modell der sieben OE-Basisprozesse. Göttingen: Haupt.

Infodrog/Fachverband Sucht (2011): Standards Kontakt- und Anlaufstellen. www.tinyurl.com/r3qzhc7, Zugriff 28.12.2019.

Lippmann, E.D. (2013): Intervision Kollegiales Coaching professionell gestalten. Berlin, Heidelberg: Springer.

Miller, W./Rollnick, S. (2015): Motivierende Gesprächsführung. Freiburg i. B.: Lambertus.

Schweizerisches Gesundheitsobservatorium (2010): OBSAN Bulletin 3/2010. Stationäre Psychiatrie der Schweiz im Wandel. www.tinyurl.com/schzt5y, Zugriff 28.12.2019.

Tietze, K.-O. (2010): Kollegiale Beratung - Problemlösungen gemeinsam entwickeln. Hamburg: Rowohlt

Wendt, W.R. (2001): Case Management im Sozial- und Gesundheitswesen. Eine Einführung. Freiburg i. B.: Lambertus. 
Open Access Dieses Kapitel wird unter der Creative Commons Namensnennung 4.0 International Lizenz (http://creativecommons.org/licenses/by/4.0/deed.de) veröffentlicht, welche die Nutzung, Vervielfältigung, Bearbeitung, Verbreitung und Wiedergabe in jeglichem Medium und Format erlaubt, sofern Sie den/die ursprünglichen Autor(en) und die Quelle ordnungsgemäß nennen, einen Link zur Creative Commons Lizenz beifügen und angeben, ob Änderungen vorgenommen wurden.

Die in diesem Kapitel enthaltenen Bilder und sonstiges Drittmaterial unterliegen ebenfalls der genannten Creative Commons Lizenz, sofern sich aus der Abbildungslegende nichts anderes ergibt. Sofern das betreffende Material nicht unter der genannten Creative Commons Lizenz steht und die betreffende Handlung nicht nach gesetzlichen Vorschriften erlaubt ist, ist für die oben aufgeführten Weiterverwendungen des Materials die Einwilligung des jeweiligen Rechteinhabers einzuholen.

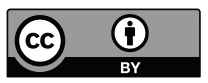

